\title{
Tumor-Suppressing STF CDNA 3 Overexpression Suppresses Renal Fibrosis by Alleviating Anoikis Resistance and Inhibiting the PI3K/Akt Pathway
}

\author{
Fei Xiao Xinghong Liu Yan Chen Huanzi Dai \\ Department of Nephrology, Daping Hospital, Army Medical University, Chongqing, China
}

\author{
Keywords \\ Renal fibrosis - Tumor-suppressing STF CDNA 3 . \\ Transforming growth factor beta $1 \cdot$ HK- 2 cells . \\ Phosphatidylinositol-4,5-bisphosphate 3-kinase/protein \\ kinase B signaling pathway
}

\begin{abstract}
Background: Myofibroblast (MF) activation is the key event of irreversible renal interstitial fibrosis. Anoikis resistance is the hallmark of active MFs, which is conferred by continuous activation of the phosphatidylinositol-4,5-bisphosphate 3-kinase (PI3K)/protein kinase B (Akt) pathway. Our previous study found that tumor-suppressing STF cDNA 3 (TSSC3) enhances the sensitivity of cells to anoikis via the PI3K/Akt pathway. Therefore, we hypothesized that TSSC3 might suppress renal interstitial fibrosis by inducing anoikis via the PI3K/Akt pathway. Methods: Cell anoikis was induced by the exogenous addition of RGD-containing peptides or by culturing cells in suspension. MFs were established by stimulating HK-2 renal tubular epithelial cells with transforming growth factor beta 1 (TGF- $\beta 1)$. Lentivirus vectors were to construct a TSSC3 overexpression cell model. The effects of TSSC3 on the anoikis, growth, migration, invasion, and contraction of MFs were determined using annexin V-fluoresce-
\end{abstract}

in isothiocyanate assays, cell counting kit-8 assays, wound healing migration assays, matrigel invasion assays, and collagen-based contraction assays. Results: The results demonstrated that TGF- $\beta 1$, simultaneous with the induction of MF differentiation, confers significant protection against anoikis-induced cell death, which could be partly reversed by treatment with the PI3K/Akt pathway inhibitor, LY294002. Moreover, overexpression of TSSC3 obviously impaired cell growth, cell migration, cell invasion, contraction, and anoikis resistance of MFs, and decreased the activity of the PI3K/Akt pathway and the production of extracellular matrix molecules, all of which could be attenuated by treatment with the $\mathrm{PI3K} /$ Akt pathway activator, 740Y-P. Taken together, this study suggested that TSSC3 attenuates the anoikis resistance and profibrogenic ability of TGF- $\beta 1$-induced MF by regulating the PI3K-Akt pathway. Conclusion: These findings provide a biological basis for further exploration of the therapeutic significance of targeting MF via TSSC3 in renal interstitial fibrosis.

() 2021 The Author(s).

Published by S. Karger AG, Basel

Fei Xiao, Xinghong Liu, and Yan Chen contributed equally to this work.
(C) 2021 The Author(s)

Published by S. Karger AG, Basel

This is an Open Access article licensed under the Creative Commons Attribution-NonCommercial-4.0 International License (CC BY-NC) (http://www.karger.com/Services/OpenAccessLicense), applicable to the online version of the article only. Usage and distribution for commercial purposes requires written permission.
Correspondence to:

Huanzi Dai, daihzxyz@163.com 


\section{Introduction}

Renal fibrosis is the common final manifestation of almost all cases of progressive CKD, regardless of the nature of the initial kidney injury (initial cause) [1]. With the development of renal fibrosis, the formation and accumulation of the extracellular matrix (ECM) is inevitable, which will lead to the loss of kidney function and eventually renal failure [2]. Myofibroblasts (MFs) are a type of activated fibroblasts, characterized by the expression of $\alpha$-smooth muscle actin ( $\alpha$-SMA), and are considered as effector cells that play a key role in the regulation of renal fibrosis [2]. In renal fibrosis and other tissue fibroses, MFs were identified as the main ECM-producing cells during fibrosis progression. Their proliferative activity also plays an important role in regulating the progression of renal fibrosis [3, 4]. Studies have demonstrated that one of the important sources of MFs in renal fibrosis is injured renal tubular epithelial cells that undergo epithelial-mesenchymal transformation induced by exposure to transforming growth factor- $\beta 1$ (TGF- $\beta 1$ ), which is a well-known and important regulator of the pathogenesis of renal fibrosis [5]. In this process, TGF- $\beta 1$ induces de novo synthesis of $\alpha$-SMA in renal tubular cells, while MFs respond to TGF- $\beta 1$ by secreting matrix molecules [6]. Accumulating evidence indicates that the number of $\alpha$-SMA-expressing MFs is the best prognostic indicator of kidney disease progression [7]. Therefore, indepth exploration of the molecular mechanism of TGF$\beta 1$-induced regulation of cell proliferation activity and functional phenotype of MFs has important clinical significance to identify therapeutic targets to offset the loss of renal function.

Anoikis, meaning the loss of "home" or "homeless," is a unique pattern of programmed cell death, which is the final result of insufficient cell matrix interaction in the microenvironment after cell separation from ECM [8]. Initially, anoikis was believed to be a physiological process that maintains tissue homeostasis in normal cells by inducing apoptosis [8]. In recent years, evidence showed that anoikis is a potentially important player in the angiogenesis and metastasis of malignant tumors [9-11]. Although the role of anoikis in tissue fibrosis has been rarely studied and remains unclear, we were interested in the observation that TGF- $\beta 1$ is capable of protecting MFs from anoikis, thus promoting the progress of lung fibrosis [12]. Therefore, verifying whether TGF- $\beta 1$ affects anoikis of MFs in renal fibrosis and exploring the underlying mechanism would deepen our understanding of the regulatory mechanism of TGF- $\beta 1$ in renal fibrosis.

TSSC3 Suppresses TGF- $\beta 1$-Induced Renal Fibrosis
Classic Mendelian genetics proposed that all paternal and maternal alleles have equal expression. However, with the progress of genetic research, it was found that the genetic imprinting of single-parent expression is also an important epigenetic regulatory mechanism for human genes $[13,14]$. Imprinted genes have been recognized to be involved in the occurrence and development of various malignant tumors. Unlike Mendel's law, a mutation in only 1 allele is required to induce dysfunction of certain imprinted genes that act as tumor suppressors, resulting in the occurrence of tumors [15-17]. TSSC3 (encoding tumor-suppressing STF cDNA 3 [TSSC3], also known as PHLDA2, BWR1C, or IPL) is located in the region 15 of the short arm of chromosome 11 and belongs to the tumor suppression region. TSSC 3 was the first confirmed apoptosis-related imprinted gene [18]. To date, most of the functional studies of TSSC 3 have focused on its role in malignant tumors. Early research indicated the absence of TSSC 3 in Wilms tumor and glioma $[18,19]$. Subsequent studies showed that TSSC 3 could inhibit the progression of osteosarcoma and explored the underlying mechanism [20-22]. Among the mechanisms by which TSSC 3 suppresses osteosarcoma, we observed that TSSC 3 , by interacting RAN-binding protein 9, could suppress the anoikis resistance of osteosarcoma cells, thus inhibiting tumor progression [23].

Bearing this in mind, we hypothesized that TSSC3 might regulate TGF- $\beta 1$-induced anoikis resistance and fibrosis progression. In the present study, we treated human renal tubular epithelial HK-2 cells with TGF- $\beta 1$ to construct an MF model, cultured HK-2 cells in ultra-low attachment culture plates or treated them with arginineglycine-aspartate (RGD)-containing peptides to induce anoikis, and transfected TSSC3-overexpressing lentiviruses into HK-2 cells to establish a cell model of TSSC3 overexpression to investigate the role of TSSC3 in TGF$\beta 1$-induced anoikis resistance and renal fibrosis progression and the underlying mechanism.

\section{Materials and Methods}

\section{Cell Culture and Treatment}

The immortalized human proximal tubular epithelial cell line HK-2 was obtained from the American Type Culture Collection (ATCC, Manassas, VA, USA) and cultured in DMEM (Gibco, Grand Island, NY, USA) and DMEM-F12 medium (Gibco) (1:1) containing $10 \%$ high-quality fetal bovine serum (Gibco), $1.80 \mathrm{~g} / \mathrm{L}$ sodium bicarbonate, $100 \mathrm{U} / \mathrm{mL}$ penicillin (Beyotime Biotechnology, Jiangsu, China), and $100 \mathrm{mg} / \mathrm{mL}$ streptomycin (Beyotime Biotechnology). Cells were cultured in a cell incubator under humid conditions at $37^{\circ} \mathrm{C}$ with $5 \% \mathrm{CO}_{2}$; the medium was refreshed 
every 2-3 days. According to the design of the experiments, certain groups of cells were treated with TGF- $\beta 1(50 \mathrm{ng} / \mathrm{mL})$, the phosphatidylinositol-4,5-bisphosphate 3-kinase $(\mathrm{PI} 3 \mathrm{~K}) /$ protein kinase $\mathrm{B}$ (Akt) signaling pathway inhibitor LY294002 $(10 \mu \mathrm{M})$, or the PI3K/ Akt signaling pathway activator $740 \mathrm{Y}-\mathrm{P}(500 \mu \mathrm{g} / \mathrm{mL})$ before the experiments.

\section{Anoikis Model Construction}

Two methods were applied to construct the anoikis model. In ultralow attachment plate method, cells were collected, centrifuged at 1,000 rpm for $5 \mathrm{~min}$, and the cell concentration was adjusted to $10^{6} / \mathrm{mL}$. The cells were inoculated into the ultralow attachment plates (Corning Inc., Corning, NY, USA) for 24 or $48 \mathrm{~h}$. In RGD method, the cells $\left(10^{6} / \mathrm{mL}\right)$ were cultured in medium containing different concentrations $(0,2$, and $4 \mathrm{mg} / \mathrm{mL})$ of RGD-containing peptides (Sigma, St. Louis, MO, USA). Cell apoptosis was detected using flow cytometry.

\section{Cell Apoptosis Assay}

For each group, $5 \times 10^{5}$ cells were inoculated in a 6-well plate and grown until the cell confluence reached $70 \%$. The cells were treated according to the experimental design and then harvested by centrifugation at $1,000 \mathrm{~g}$ for $5 \mathrm{~min}$. The supernatant was discarded, and $195 \mu \mathrm{L}$ of annexin V-fluorescein isothiocyanate (FITC)-binding solution was added to gently resuspend the cells. Then, $5 \mu \mathrm{L}$ of annexin V-FITC $(20 \mu \mathrm{g} / \mathrm{mL}$; BD Biosciences, San Jose, CA, USA) was added, and the cells were cultured for $10 \mathrm{~min}$ in the dark. After centrifuging and discarding the supernatant, 190 $\mu \mathrm{L}$ of annexin V-FITC binding solution and $10 \mu \mathrm{L}$ of propidium iodide staining solution $(50 \mu \mathrm{g} / \mathrm{mL})$ were added, and the cells were cultured in an ice bath. Then, the cell apoptosis rate was detected and calculated using a FACSCalibur flow cytometer (BD Biosciences).

\section{Quantitative Real-Time Reverse Transcription PCR}

The TRIzol reagent (Sigma) was used to extract RNA from HK-2 cells. The RNA quality was evaluated using a Nanodrop 2000/2000C spectrophotometer (Thermo Fisher Scientific, Waltham, MA, USA) according to the manufacturer's instructions. A Promega M-MLV kit (Promega, Madison, WI, USA) was used to reverse transcribe the RNA $(2.0 \mu \mathrm{g})$ to cDNA. Two-step qPCR was performed on a realtime fluorescence quantitative PCR instrument (Eppendorf, Hamburg, Germany) using specific primers with an SYBR Green Master Mix Kit (Western Biotechnology, Perth, Australia). A melting curve was drawn, and the relative quantitative gene expression was analyzed using the $2^{-\triangle \Delta C t}$ method. GAPDH (glyceraldehyde-3-phosphate dehydrogenase) acts as the internal control. The specific upstream and downstream primer sequences for the PCR reaction were as follows - gene name, forward primer sequence, reverse primer sequence: $\alpha$-SMA, $5^{\prime}$-TGTACCCTGGGATCGCTGAC- $3^{\prime}, 5^{\prime}$-CTCGTCGTACTCCTGCTTGG- ${ }^{\prime}$; villin, 5' $^{\prime}$-TGTCAAGGGCAAGAGGAACG-3', 5'-CCCCTGAGTCTCTCCATACGG-3'; actin, 5'-TGACGTGGACATCCGCAAAG-3', 5' ${ }^{\prime}$-CTGGAAGGTGGACAGCGAGG-3'; TSSC $3,5^{\prime}$ - TCCAGCTATGGAAGAAGAAGC-3', $5^{\prime}$-GTGGTGACGATGGTGAAGTACA-3'; matrix metalloproteinase-2 (MMP-2), 5' ${ }^{\prime}$-TTTGACGGTAAGGACGGACTC-3', $5^{\prime}$-TACTCCCCATCGGCGTTC-3'; secreted protein acidic and cysteine rich (SPARC), 5'-AGGAAACCGAAGAGGAGG-3', $5^{\prime}$-GCAAAGAAGTGGCAGGAA-3'; transglutaminase 2 (TG2), $5^{\prime}$-GCCCTGCGTAAGGACATATT-3', 5'-GTGCAGGGTCC-
GAGGT-3'; collagen type I alpha 1 chain (COL1A1), $5^{\prime}$-GTGCGATGACGTGATCTGTGA-3', 5'-GTTTCTTGGTCGGTGGGTG-3'; collagen type III alpha 1 chain (COL3A1), $5^{\prime}$-TGCTCGGGGTAATGACGG-3', 5'-GCACCATTTGAACCAGGAGAC- $3^{\prime}$; fibronectin, $5^{\prime}$-ACAGACCTATCCAAGCTCAAGTG-3', $5^{\prime}$-AAATGTGAGATGGCTGTGGTG-3'; ${ }^{\prime \prime}$ and GAPDH, 5' ${ }^{\prime}$-TGACGTGGACATCCGCAAAG-3' ${ }^{\prime}$ ' '-CTGGAAGGTGGACAGCGAGG-3'.

\section{Western Blotting Assay}

After the cells were fully lysed in ice-cold radioimmunoprecipitation assay buffer (Millipore, Billerica, MA, USA), they were centrifuged at $12,000 \mathrm{rpm}, 4^{\circ} \mathrm{C}$ for $15 \mathrm{~min}$, to collect total proteins. The protein concentration was detected using a bicinchoninic acid (BCA) Protein Assay Kit (BCA Reagent A:BCA Reagent B = 100: 1; HyClone-Pierce, Logan, UT, USA). Protein (20 $\mu$ g per lane) was separated using 10\% SDS-PAGE (Amresco, Solon, OH, USA) and then transferred onto PVDF (Bio-Rad, Hercules, CA, USA) membranes at $4^{\circ} \mathrm{C}$. After blocking with $5 \%$ skim milk in Tris-buffered saline-Tween-20 solution, the membranes were incubated at $4^{\circ} \mathrm{C}$ overnight with primary antibodies: $\alpha$-SMA (ab5694; Abcam, Cambridge, MA, USA), villin (Abcam, 1:500), phosphorylated (p)PI3K-P85 (ab182651; Abcam), PI3K-P85 (ab86714; Abcam), pAkt-ser473 (1:500; Abcam), Akt (ab18785; Abcam), cleaved caspase 3 (1:500; Abcam), pro-caspase 3 (1:500; Abcam), TSSC3 (ab173039; Abcam), BCL2-associated X, apoptosis regulator (Bax) (ab53154; Abcam), BCL2 apoptosis regulator (Bcl-2) (ab32124; Abcam), cleaved caspase 9 (ab2324; Abcam), procaspase 9 (ab32539; Abcam), cytochrome (C) (ab133504; Abcam), MMP-2 (ab97779; Abcam), MMP-9 (ab76003; Abcam), COL1A1 (ab6308; Abcam), COL3A1 (ab7778; Abcam), fibronectin (ab32419; Abcam), SPARC (ab225716; Abcam), and GAPDH (1:1,000; Abcam). After washing with Tris-buffered saline-Tween-20 3 times, the membranes were further incubated with secondary antibodies (goat anti-rabbit IgG; Sigma) for $1.5 \mathrm{~h}$ at room temperature. Immunoreactive protein bands were visualized using an ECL-PLUS Kit (Pierce Biotechnology, Rockford, IL, USA) and gray analysis of the bands was performed using Image J software (NIH, Bethesda, MD, USA).

\section{Cell Counting Kit-8 Assay}

The cell suspension $\left(100 \mu \mathrm{L} ; 1 \times 10^{5} / \mathrm{mL}\right)$ was seeded into each well of a 96-well plate in triplicate and cultured at $37^{\circ} \mathrm{C}$ with $5 \%$ $\mathrm{CO}_{2}$. For each group, after culture for $24 \mathrm{~h}, 10 \mu \mathrm{L}$ of Cell Counting Kit-8 (CCK-8) reagent (Sigma) was added into each well and cultured for $4 \mathrm{~h}$ in an incubator until the visual color conversion occurred. The optical density at $450 \mathrm{~nm}$ was measured using a microplate reader (Thermo Fisher Scientific), and the cell inhibition rate was calculated.

\section{TSSC3-Overexpressing Lentivirus Construction and}

Transfection

TSSC3-overexpressing lentivirus was purchased from Invabio (Shanghai, China). An HK-2 cell suspension $\left(5 \times 10^{5} / \mathrm{mL}\right)$ was inoculated into a 24 -well culture plate with $100 \mu \mathrm{L}$ of culture medium per well. Cells were cultured until the cell confluence reached $70 \%$, and then, the TSSC3-overexpressing lentivirus $\left(1 \times 10^{8} \mathrm{TU} / \mathrm{mL}\right)$ and polybrene $(8 \mu \mathrm{g} / \mathrm{mL})$ were added into each well and cultured for $72 \mathrm{~h}$. The transfected cells were screened using puromycin (100 $\mathrm{ng} / \mathrm{mL}$ ). HK-2 cells successfully transfected with the TSSC3-overexpressing lentivirus were maintained under a low concentration 
of puromycin. The normal cell control group and empty lentivirus transfection group were set as controls.

\section{Wound Healing Assay}

HK-2 cells were seeded into a 6-well dish (Costar, Richmond, VA, USA) with $1 \times 10^{5} / \mathrm{mL}$ cells per well and cultured in a $37^{\circ} \mathrm{C}$ and $5 \% \mathrm{CO}_{2}$ incubator for $48 \mathrm{~h}$. For each group, when the cell confluence reached $80 \%$, wounds were made across the central part of each cell layer using a $200-\mu \mathrm{L}$ pipette tip (Axygen Biosciences, Union City, CA, USA). Free cells were gently washed off using PBS 3 times, and medium without fetal bovine serum was added. The cells were treated according to the experimental scheme and further cultured for $0,6,24$, or $30 \mathrm{~h}$. Photographs were taken under a fluorescence microscope $(\times 100$; Olympus, Tokyo, Japan $)$, and the migration rate was calculated.

\section{Transwell Assay}

HK-2 cell suspension $\left(250 \mu \mathrm{L}\right.$ of $\left.1 \times 10^{5} \mathrm{cell} / \mathrm{mL}\right)$ was seeded into the upper chamber of a 24-well Transwell migration insert (Costar). The membrane pore diameter of the Transwell cell was $12 \mu \mathrm{m}$ and was covered by Matrigel (BD Biosciences). Cell medium $(200 \mu \mathrm{L})$ was added into the lower chamber and the whole insert was cultured. Next, the Transwell was taken out, and the remaining cells were gently wiped off with cotton balls. The lower surface was soaked in $70 \%$ methanol, fixed for $60 \mathrm{~min}$, and stained with $0.1 \%$ crystal violet (Sigma). Images were obtained under a microscope at $\times 100$ magnification, and the number of cells under the surface of membrane was calculated using the average value of 5 visual fields.

\section{Collagen Gel Contraction Assay}

The collagen gel contraction assay was performed using a Collagen-Based Contraction Assay Kit (Cell Biolabs Inc., San Diego, CA, USA). Cold collagen gel solution was prepared by mixing 9.54 $\mathrm{mL}$ of collagen solution, $2.46 \mathrm{~mL}$ of $5 \times \mathrm{PBS}$, and $340 \mu \mathrm{L}$ of the corresponding neutralization solution on ice. Then, a cell suspension at $5 \times 10^{5} / \mathrm{mL}$ (treated with or without TGF- $\beta 1[50 \mathrm{ng} / \mathrm{mL}]$ ) was added into $4 \times$ cold collagen gel solution and mixed. Then, $0.5 \mathrm{~mL}$ of the cell-collagen gel mixture was seeded into a 24 -well plate and cultured for $1 \mathrm{~h}$. After collagen polymerization, $1.0 \mathrm{~mL}$ of medium was added to each well and further cultured at $37^{\circ} \mathrm{C}$ in a $5 \% \mathrm{CO}_{2}$ atmosphere for $24 \mathrm{~h}$. The cells were treated with $10 \mathrm{~mm}$ bubaline diaphragm matrix, and the collagen gel was gently released from the side of the dish with sterile scrapers. The collagen gel was observed under an inverted microscope at $\times 100$ and $\times 200$, and the area of each gel was measured using NIH ImageJ software.

\section{Statistical Analysis}

All experiments were performed in triplicate, and data are shown as the mean $\pm \mathrm{SD}$. The significant differences between groups were determined using a 2-tailed Student's $t$ test (2 groups) or 1-way ANOVA (3 groups or more). Statistical significance was analyzed by SPSS 22.0 (IBM, Armonk, NY, USA), and $p$ values $<0.05$ were considered statistically significant. Graphs were drawn using GraphPad Prism 7 (GraphPad Software Inc., La Jolla, CA, USA).

\section{Results}

Loss of Cell Adhesion or Treatment with Exogenous RGD-Containing Peptides Induces HK-2 Cell Anoikis

In this study, we utilized 2 experimental models to induce anoikis of HK-2 cells: (1) culturing cells in ultralow attachment culture plates to mechanically detach cells and maintain the cells in suspension for specified periods of time (model 1) [24] and (2) competitive interruption of integrin-ECM interactions by the addition of soluble peptides containing an RGD motif (model 2) [12]. The formation of multicellular aggregates after adherence to the ECM by HK-2 cells was abrogated by culturing under suspension conditions in ultralow attachment plates. As the culture continued, a significant time-dependent decrease in the size of cell aggregates that survived in the ultralow attachment culture plates was observed (Fig. 1a), suggesting that HK-2 cells underwent anoikis after culture under suspended conditions. To quantify apoptosis, we performed annexin V/PI staining and flow cytometry. Cells in suspension (group 0U) demonstrated a time-dependent increase in the rates of apoptosis, with apoptosis being observed within $24 \mathrm{~h}$ of suspension and a further increase noted at $48 \mathrm{~h}$ of suspension (Fig. 1b). Next, we assessed the ability of soluble RGD-containing peptides to induce anoikis of HK-2 cells. Prominent dose-dependent morphological changes associated with cell rounding, withdrawal of cellular foci, and surface detachment leading to anoikis were observed in RGD-treated cells (Fig. 1c). Moreover, upon treatment with RGD-containing peptides, a dose-dependent increase in cell apoptosis was also observed (Fig. 1d).

\section{TGF- $\beta 1$ Induces the Conversion of HK-2 Cells into MFs}

Treatment with TGF- $\beta 1$ was used to induce activation and conversion of tubular epithelial cells into an MF phenotype. Exposure of HK-2 cells to $50 \mathrm{ng} / \mathrm{mL}$ TGF- $\beta 1$ caused time-dependent morphological changes under phase-contrast microscopy, with cells cultured for 7 day developing marked hypertrophy, becoming elongated, and losing their cobblestone growth pattern (Fig. 2a). We performed time course experiments using $50 \mathrm{ng} / \mathrm{mL}$ TGF- $\beta 1$ to examine $\alpha$-SMA as a robust marker of MF differentiation and villin as an epithelial marker in HK-2 cells using qRT-PCR and Western blotting assays. As shown in Figure $2 b, c$, treatment with TGF- $\beta 1$ increased the expression of $\alpha$-SMA significantly and decreased the expression of villin in a time-dependent 


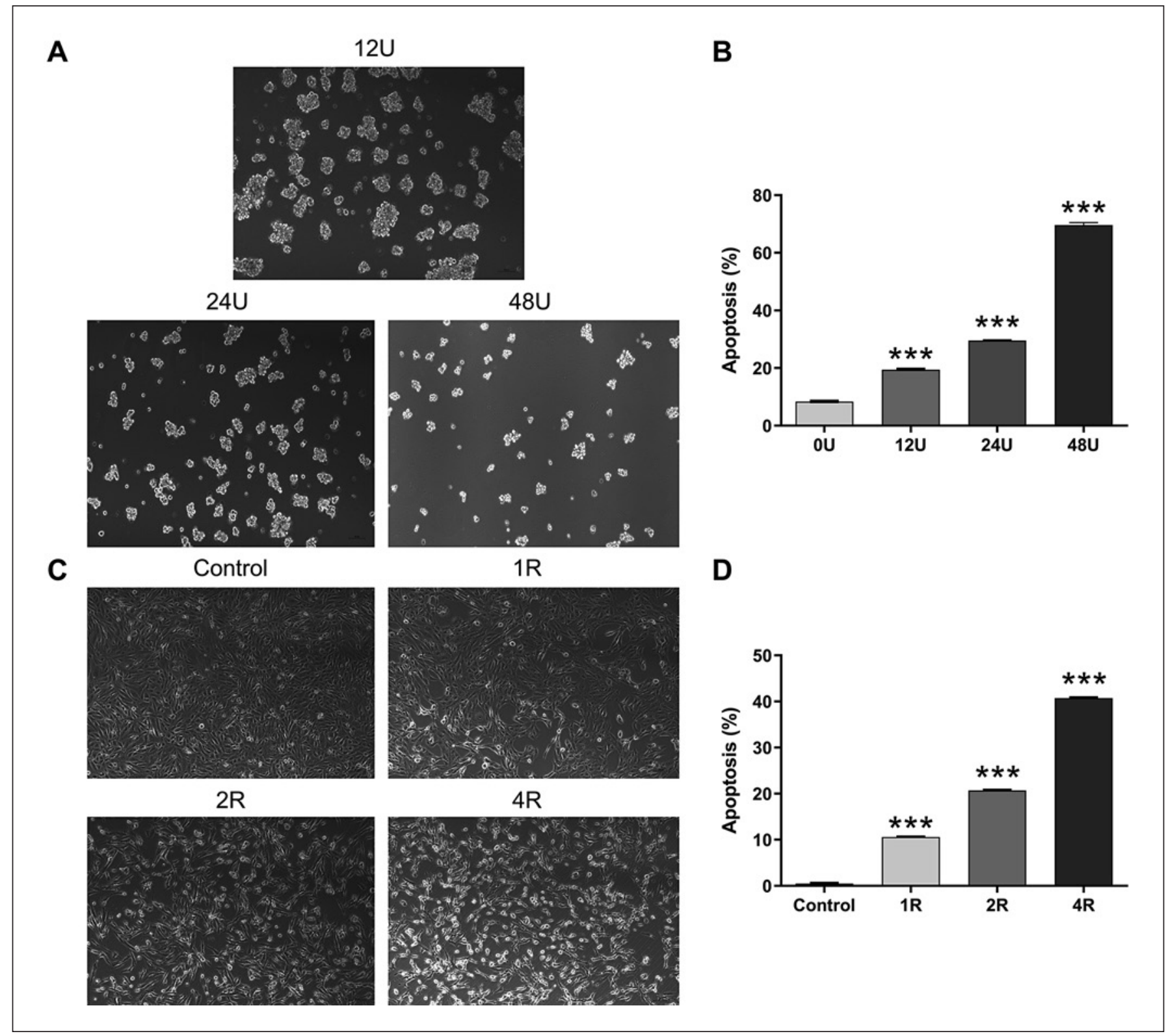

Fig. 1. Construction of the anoikis model. A An anoikis model using HK-2 cells was constructed by culturing them on ultralow attachment culture plates, and a microscope was used to observe the cell morphology. B Cell apoptosis of HK-2 cells cultured on ultralow attachment culture plates was evaluated by flow cytometry. Control: HK-2 cells cultured on normal plates; 0/12/24/48U: HK-2 cells cultured on ultralow attachment culture plates for $0,12,24$,

manner. These results indicated that exposure of HK-2 cells to $50 \mathrm{ng} / \mathrm{mL}$ TGF- $\beta 1$ could induce a time-dependent conversion of epithelial cells to MFs. Treatment with $50 \mathrm{ng} / \mathrm{mL}$ TGF- $\beta 1$ for $72 \mathrm{~h}$ was used in subsequent experiments. or 48 h. C Treatment of HK-2 cells with RGD-containing peptides was used to induce anoikis, and the microscope was used to observe the cell morphology. D Cell apoptosis of HK-2 cells treated with or without RGD was evaluated. Control: HK-2 cells with no treatment. 1/2/4R: HK-2 cells treated with 1, 2, or $4 \mathrm{mg} / \mathrm{mL}$ RGD. Data are shown as the mean with the SD. ${ }^{* * *} p<0.001$. RGD, arginine-glycine-aspartate.

\section{TGF- $\beta 1$ Protects Renal MFs from Anoikis}

To investigate the effects of TGF- $\beta 1$ treatment on anoikis of renal MFs, HK-2 cells were pretreated with or without TGF- $\beta 1$ and then cultured under suspension conditions. The detection of cell apoptosis by flow cytom- 
A

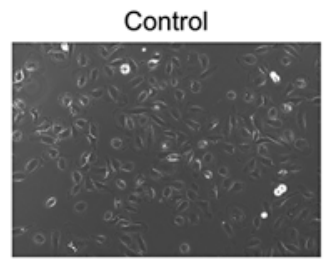

B

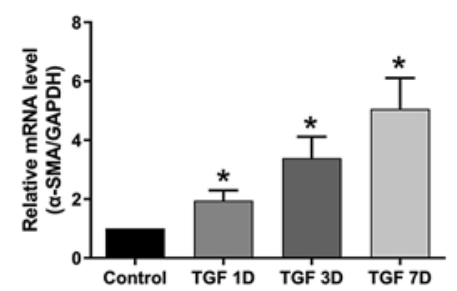

D

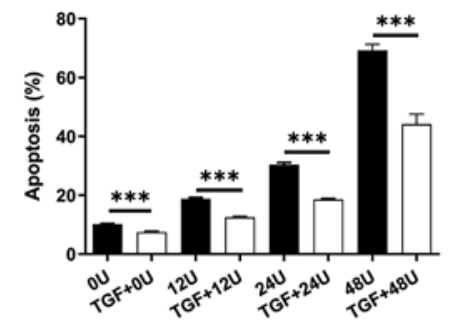

$\mathbf{F}$

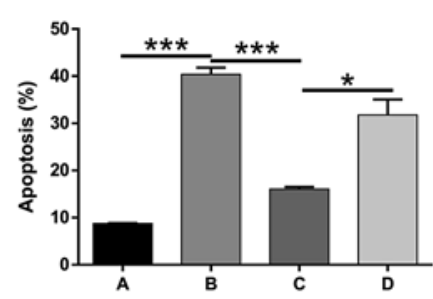

A: Control; B: anoikis (4R); C: TGF+4R; D: TGF+LY294002+4R

TGF 1D

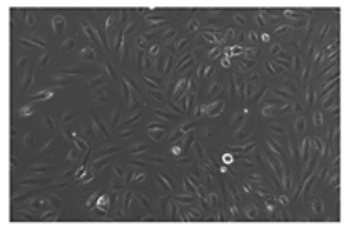

G
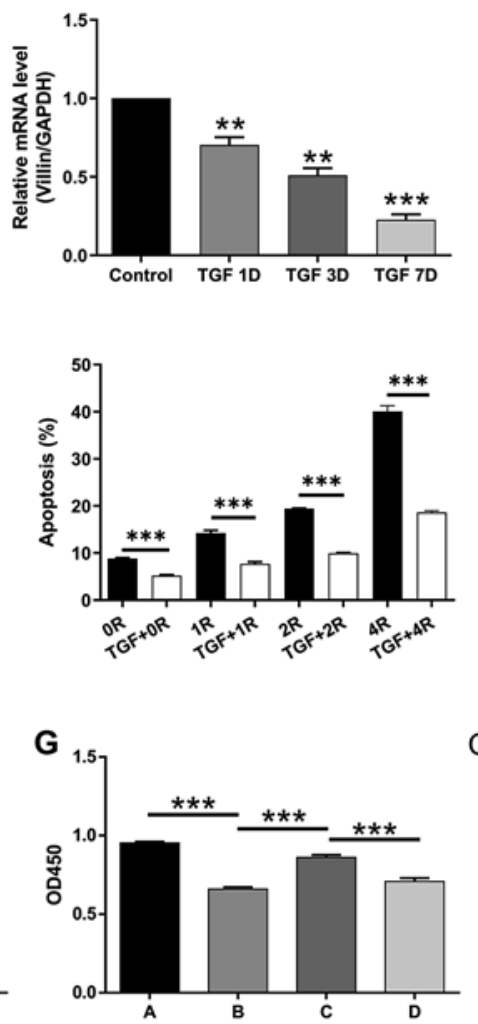

Cleaved Caspase3

Pro-Caspase3

GAPDH

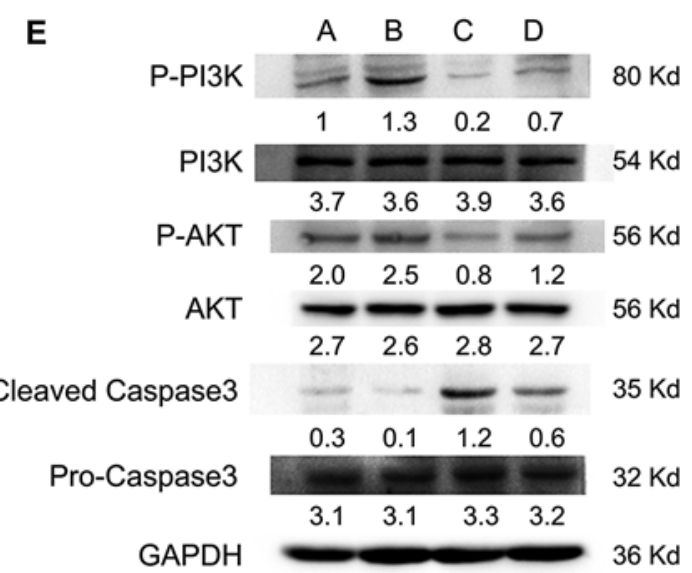

A: Control; B: TGF; C: anoikis (4R); D: TGF+4R;
Fig. 2. TGF- $\beta 1$ protects renal MFs from anoikis via the PI3K/Akt pathway. A Cell morphology of HK-2 cells treated with TGF- $\beta 1$ (50 ng/mL) for different times was recorded under a microscope. B, C qRT-PCR and Western blotting assays were performed to detect the mRNA (B) and protein (C) expression of $\alpha$-SMA and villin in HK-2 cells treated with TGF- $\beta 1(50 \mathrm{ng} / \mathrm{mL})$ for different times. D Flow cytometry was used to assess cell apoptosis of HK-2 cells in the different groups. E The expression of proteins in the PI3K/ Akt signaling pathway in different groups of HK-2 cells. F Flow cytometry was used to assess the cell apoptosis of HK-2 cells in different groups. G CCK-8 assay was used to evaluate cell prolifera-

etry showed that the average apoptosis frequencies of HK-2 cells cultured in ultralow attachment plates for 0 , 12,24 , and $48 \mathrm{~h}$ were $10.2,118.9,30.4$, and $69.2 \%$, respectively, while the apoptosis rates in MFs cultured in ultralow attachment plates for $0,12,24$, and $48 \mathrm{~h}$ were 7.5 , tion of HK-2 cells in the different groups. The quantification of immunoreactive proteins on the Western blots was normalized using the level of GAPDH. Control: no treatment; TGF 1/3/7D: treatment with TGF- $\beta 1(50 \mathrm{ng} / \mathrm{mL})$ for 1,3 , or 7 days; $0 / 12 / 24 / 48 \mathrm{U}$ : culture on ultralow attachment culture plates for $0,12,24$, or $48 \mathrm{~h}$; 0/1/2/4R: treatment with $0,1,2$, or $4 \mathrm{mg} / \mathrm{mL}$ RGD; and LY294002: treatment with LY294002 $(5 \mu \mathrm{M})$. Data are shown as the mean with the SD. ${ }^{*} p<0.05,{ }^{* *} p<0.01,{ }^{* * *} p<0.001$. TGF- $\beta 1$, transforming growth factor beta 1; MF, myofibroblast; $\alpha$-SMA, $\alpha$-smooth muscle actin; CCK-8, Cell Counting Kit-8; GAPDH, glyceraldehyde3-phosphate dehydrogenase; RGD, arginine-glycine-aspartate. that TGF- $\beta 1$ treatment induced distinct anoikis resistance. Similarly, we demonstrated that TGF- $\beta 1$-treated HK-2 cells were resistant to anoikis, as evidenced by lower rates of apoptosis with $2 \mathrm{mg} / \mathrm{mL}$ RGD treatment (19.4 
vs. $10.0 \%)$ and more significantly reduced apoptosis with $4 \mathrm{mg} / \mathrm{mL}$ RGD treatment (40.4 vs. $18.6 \%$ ) compared with that in HK-2 cells (Fig. 2d). Altogether, these results demonstrated that TGF- $\beta 1$, simultaneous with the induction of MF differentiation, confers significant protection from anoikis-induced cell death.

TGF- $\beta 1$-Induced MF Anoikis Resistance and Viability Are Regulated by the PI3K/Akt Pathway

To investigate the potential role of the PI3K/Akt pathway in TGF- $\beta 1$-induced anoikis resistance of HK- 2 cells, the activation of PI3K/Akt pathway was detected in HK-2 cells treated with TGF- $\beta 1$ and/or RGD $(4 \mathrm{mg} / \mathrm{mL})$. As shown in Figure 2e, treatment of HK-2 cells with RGD decreased the levels of p-PI3K-P85 and p-Ser ${ }^{473}$ AKT and increased the levels of cleaved caspase 3. Stimulation with TGF- $\beta 1$ augmented the levels of p-PI3K-P85 and phospho-Ser ${ }^{473}$ AKT and reduced the level of cleaved caspase 3 in cells, with or without RGD treatment. Contrastingly, RGD-treated cells demonstrated increased cell apoptosis and decreased cell proliferation, as confirmed by annexin V/PI and CCK- 8 assays, and these effects were significantly attenuated by simultaneous treatment with TGF- $\beta 1$ (Fig. 2f). To further confirm the involvement of the PI3K/ Akt pathway, LY294002 (10 $\mu \mathrm{M})$, a pharmacological inhibitor of the PI3K/Akt pathway, was utilized. Blockade of PI3K/Akt attenuated the protective effect of TGF- $\beta 1$ on RGD-induced apoptosis significantly (Fig. $2 \mathrm{~g}$ ) as well as the promotive effect of TGF- $\beta 1$ on the RGD-induced acceleration of cell growth. These results suggested that activation of the PI3K/Akt pathway is essential for the resistanceagainstanoikis acquiredbyTGF- $\beta 1$-differentiated MFs.

TSSC3 Attenuated Anoikis Resistance of TGF- $\beta 1$ -

Induced MFs via the PI3K/Akt Pathway

To explore whether TSSC 3 has a role in anoikis resistance and cell viability of TGF- $\beta 1$-induced MFs, we generated an HK-2 cell line that stably overexpressed TSSC 3 (TSSC3-OE) and matching control cells (OE-NC). The overexpression of TSSC 3 in HK-2 cells was confirmed through qRT-PCR and Western blotting assays (Fig. 3a, bb). Subsequently, the effects of TSSC 3 overexpression on anoikis or TGF- $\beta 1$-induced anoikis resistance of MFs were evaluated based on the anoikis models. As shown in Figure $3 c, d-d$, regardless of the anoikis model used, TSSC 3 overexpression could not only significantly further enhance the apoptosis of HK-2 cells but also attenuated the anoikis resistance of TGF- $\beta 1$-induced MFs. TSSC3 overexpression accelerated HK-2 cell prolifera- tion and alleviated the inhibitory effects of TGF- $\beta 1$ treatment on cell growth (Fig. 3e). These results demonstrated that TSSC3 upregulation markedly reduced the anoikis resistance and cell growth of TGF- $\beta 1$-induced MFs. We subsequently analyzed the expression of mitochondriaassociated proapoptotic and antiapoptotic proteins using Western blotting. As shown in Figure 3f-h, TGF- $\beta 1$ treatment induced decreased levels of apoptotic Bax and increased levels of antiapoptotic Bcl-2, which resulted in a dramatic decrease in the $\mathrm{Bax} / \mathrm{Bcl}-2$ ratio and cytochrome $\mathrm{c}$ in the cytosolic fraction, whereas upregulation of TSSC3 expression markedly increased the $\mathrm{Bax} / \mathrm{Bcl}-2$ ratio and cytochrome $c$ release from the mitochondria in MFs. Furthermore, TSSC3 overexpression facilitated caspase cascade activation significantly by upregulating the levels of cleaved caspase 9 and cleaved caspase 3, which were inhibited in TGF- $\beta 1$-induced MFs (Fig. 3f, h). To further reveal the involvement of the PI3K/Akt pathway, we performed Western blotting analysis, which showed that TSSC3 upregulation markedly reduced the TGF- $\beta 1$ induced increase in the levels of the p85 subunit of PI3K and Akt phosphorylation in model 2. Moreover, we found that exposure of TSSC3-overexpressing MFs to $500 \mu \mathrm{g} /$ $\mathrm{mL} 740 \mathrm{Y}-\mathrm{P}$, a potent stimulator of PI3K activity, for $24 \mathrm{~h}$, restored the levels of phosphorylated PI3K and Akt and abrogated the activation of the mitochondria-initiated apoptotic pathway, as evidenced by a decreased Bax/Bcl2 ratio, reduced cytosolic cytochrome $c$ expression, and decreased caspase cascade activation (Fig. 3f-i), thereby partially reversing TSSC3-attenuated anoikis resistance (Fig. 3j). Taken together, we concluded that TSSC3 attenuated mitochondria-associated anoikis resistance of TGF- $\beta 1$-induced MFs by downregulating the PI3K-Akt signaling pathway.

\section{TSSC3 Suppressed Migration, Invasion, and MMP}

Secretion of TGF- $\beta 1$-Induced MFs in a PI3K/AktDependent Manner

An important component of MF function is the acquisition of migratory and invasive characteristics. Therefore, the effects of TGF- $\beta 1$ treatment and TSSC 3 overexpression on cell migration and invasion were evaluated using wound healing and Transwell assays, respectively. The results demonstrated that TGF- $\beta 1$ treatment could significantly enhance the migration and invasion of HK-2 cells, which also supported the TGF- $\beta 1$-induced differentiation of MFs from HK-2 cells (Fig. 4a, b). Moreover, the TGF- $\beta 1$-induced enhancement of cell migration and invasion could be alleviated by TSSC3 overexpression (Fig. 4a, b). Additionally, as shown in Figure $4 c-d$, we 


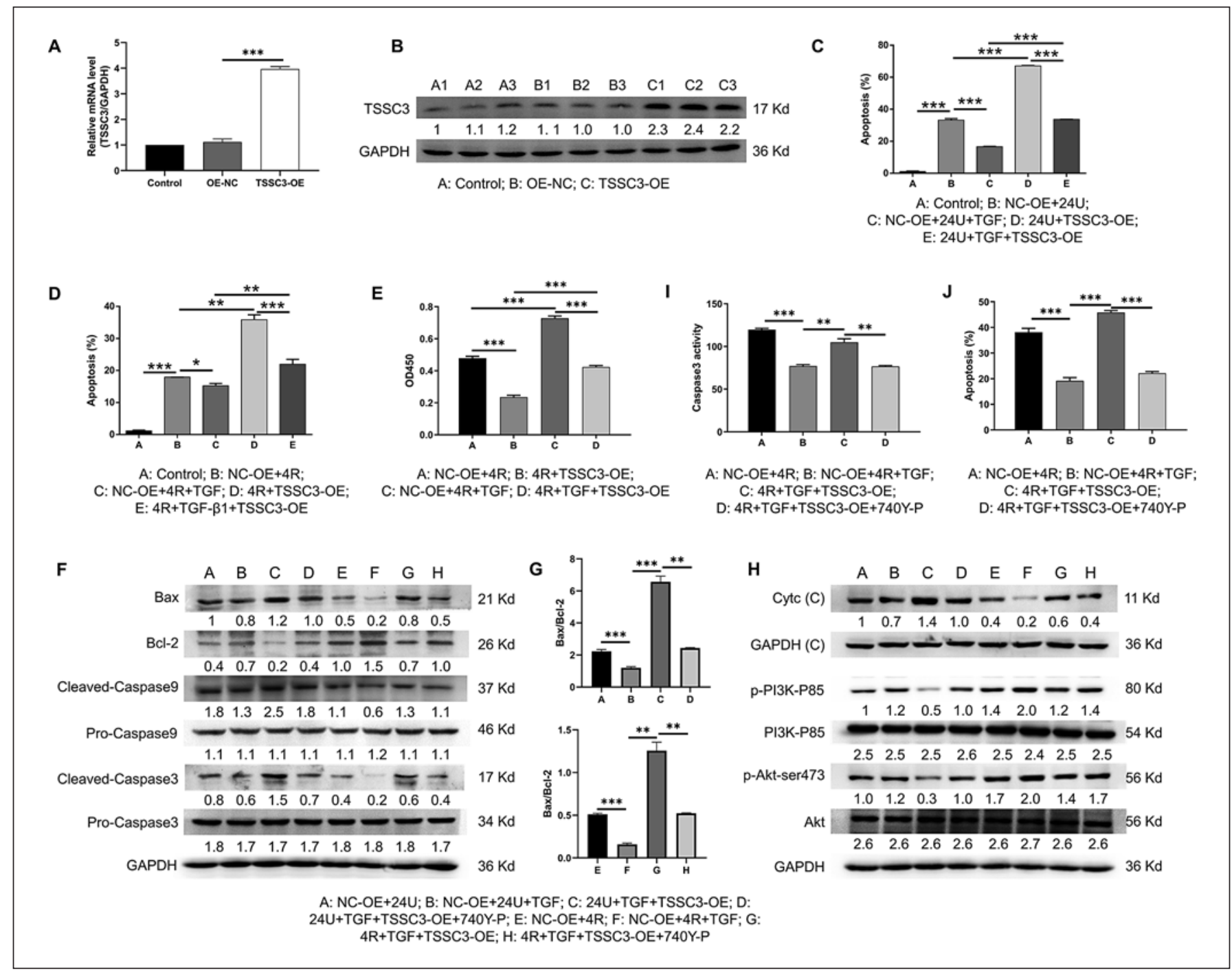

Fig. 3. TSSC 3 attenuated anoikis resistance of TGF- $\beta 1$-induced MFs via the PI3K/Akt pathway. A, B The construction of TSSC3 overexpression cell model was confirmed by detecting TSSC3 expression using qRT-PCR (A) and Western blotting (B). C, D The effects of TGF- $\beta 1$ treatment and TSSC3 overexpression on cell apoptosis were evaluated in the anoikis model induced by ultralow attachment culture plates (C) or RGD treatment (D). E The effects of TGF- $\beta 1$ treatment and TSSC 3 overexpression on cell growth were examined using CCK- 8 assays in the anoikis model induced by RGD treatment. $\mathbf{F}-\mathbf{H}$ The effects of TGF- $\beta 1$ treatment and TSSC3 overexpression on expression of apoptosis-related or PI3K/ Akt pathway-related proteins and the $\mathrm{Bax} / \mathrm{Bcl}-2$ ratio $(\mathbf{G})$ were detected by Western blotting in the anoikis model. $\mathbf{I}$, $\mathbf{J}$ The effects of TGF- $\beta 1$ treatment and TSSC 3 overexpression on caspase 3 activ-

observed that TGF- $\beta 1$-activated MFs expressed upregulated mRNA and protein levels of MMP-2 and MMP-9, which was markedly reversed by TSSC3 overexpression. ity and cell apoptosis were evaluated in the anoikis model induced by RGD treatment. The quantification of immunoreactive proteins on the Western blots was normalized using the level of GAPDH. Control: no treatment; OE-NC: treatment with negative control plasmids; TSSC3-OE: treatment with TSSC3-overexpressing plasmids; $24 \mathrm{U}$ : treatment with ultralow attachment culture plates for $24 \mathrm{~h}$; $4 \mathrm{R}$ : treatment with $4 \mathrm{mg} / \mathrm{mL}$ RGD; TGF: treatment with $50 \mathrm{ng} / \mathrm{mL}$ TGF- $\beta 1$; and $740 \mathrm{Y}-\mathrm{P}$ : treatment with $500 \mu \mathrm{g} / \mathrm{mL} 740 \mathrm{Y}-$ P. Data are shown as the mean with the SD. ${ }^{*} p<0.05,{ }^{* *} p<0.01$, ${ }^{* * *} p<0.001$. TSSC3, tumor-suppressing STF cDNA 3; TGF- $\beta 1$, transforming growth factor beta 1; MF, myofibroblast; CCK-8, Cell Counting Kit-8; GAPDH, glyceraldehyde-3-phosphate dehydrogenase; RGD, arginine-glycine-aspartate. 


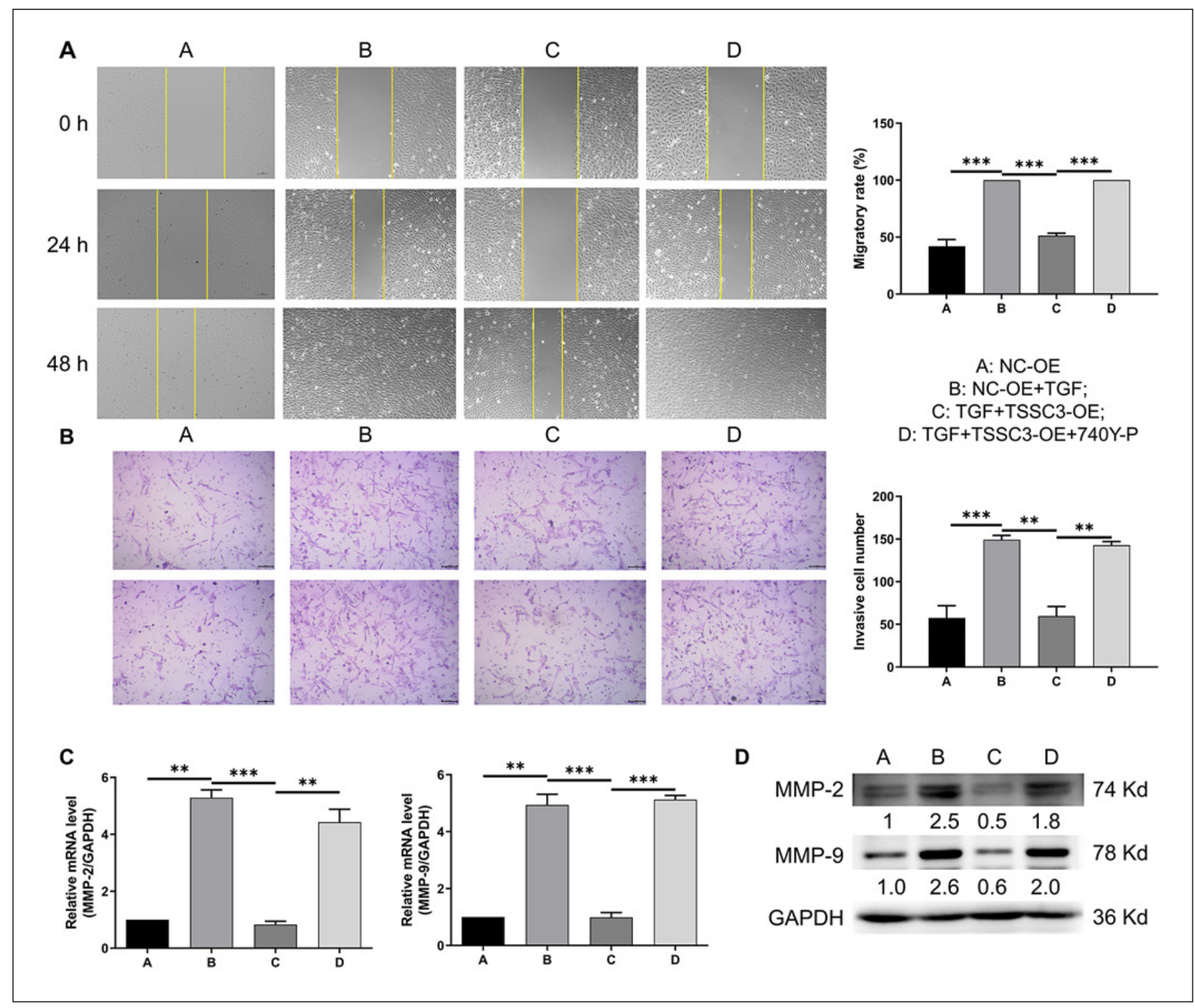

Fig. 4. TSSC 3 suppressed migration, invasion, and MMP secretion of TGF- $\beta 1$-induced MFs in a PI3K/Akt-dependent manner. A A wound healing assay was used to assess cell migration of HK-2 cell in different groups. B A Transwell assay was used to assess cell invasion of HK-2 cells in the different groups. C, D qRT-PCR and Western blotting assays were used to detect the mRNA (C) and protein (D) expression of MMP-2 and MMP-9 in HK-2 cells of the different groups. The quantification of immunoreactive proteins

The results showed that PI3K activation could partly abrogate these TSSC3-induced effects in TGF- $\beta 1$-induced MFs (Fig. 4). Taken together, these data strongly suggested that TSSC3-suppressed migration and invasion of TGF- $\beta 1$-induced MFs is dependent on activation of the PI3K/Akt pathway. on the Western blots was normalized using the level of GAPDH. OE-NC: treatment with negative control plasmids; TSSC3-OE: treatment with TSSC3-overexpressing plasmids; TGF: treatment with $50 \mathrm{ng} / \mathrm{mL}$ TGF- $\beta 1$; and 740Y-P: Treatment with $500 \mu \mathrm{g} / \mathrm{mL}$ $740 \mathrm{Y}-\mathrm{P}$. Data are shown as the mean with the SD. ${ }^{*} p<0.05,{ }^{* *} p<$ $0.01,{ }^{* *} p<0.001$. TSSC3, tumor-suppressing STF cDNA 3; MMP, matrix metalloproteinase; TGF- $\beta 1$, transforming growth factor beta 1 ; MF, myofibroblast.

TSSC3 Inhibited the Contractile Force and the ECM Production and Synthesis of TGF- $\beta 1$-Induced MFs via the PI3K/Akt Pathway

A key feature of MFs is their contractile force generation, which is aided by the increased $\alpha$-SMA expression that occurs in response to active TGF- $\beta 1$. First, we found that 


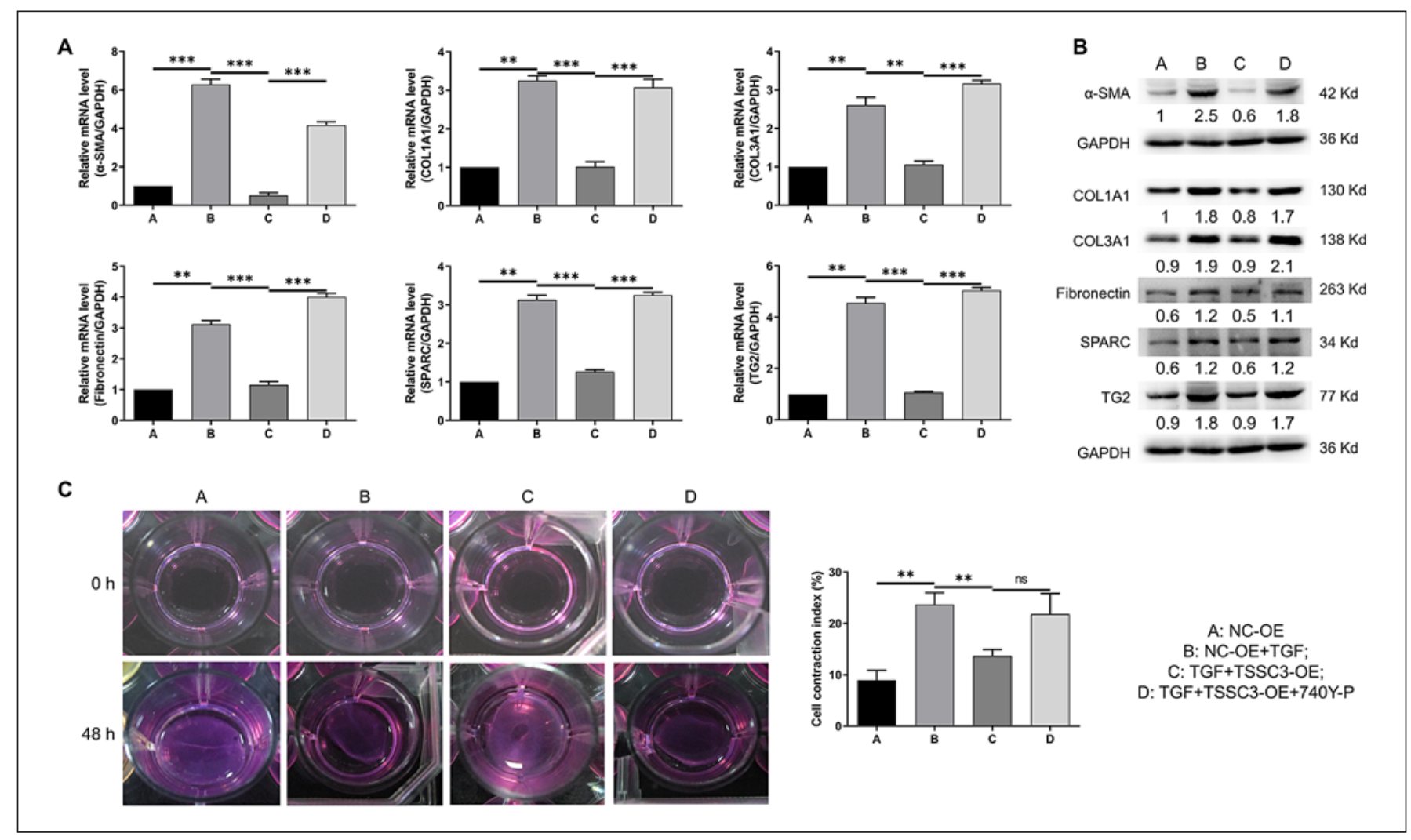

Fig. 5. TSSC3 inhibited the contractile force and the ECM production/synthesis of TGF- $\beta 1$-induced MFs via the PI3K/Akt pathway. A, B The effects of TGF- $\beta 1$ treatment, TSSC3 overexpression, and $740 \mathrm{Y}-\mathrm{P}$ treatment on the $\mathrm{mRNA}$ and protein expression of $\alpha$-SMA, COL1A1, COL3A1, fibronectin, SPARC, and TG2 was detected by qRT-PCR (A) and Western blotting assays (B), respectively. C A $3 \mathrm{D}$ collagen type I gel contraction assay was performed to show the effects of TGF- $\beta 1$ treatment, TSSC3 overexpression, and 740Y-P treatment on cell contraction. The quantification of immunoreac-

TGF- $\beta 1$ induced significant upregulation of $\alpha$-SMA mRNA and protein expression, which was partially reversed by TSSC 3 overexpression (Fig. 5a, b). A 3D collagen type I gel contraction assay was performed, which showed that TGF- $\beta 1$ treatment caused a significant gel contraction to $75.3 \%$ of the initial area, being $9.8 \%$ below the median surface of the NC-OE group (Fig. 5c). By contrast, ectopic expression of TSSC 3 completely blocked the TGF- $\beta$-induced contraction, with a median gel area of $93.85 \%$, increasing the gel area by $18.54 \%$ compared with that in the TGF$\beta 1+$ TSSC3-OE group (Fig. $5 \mathrm{c}$ ). We also tested the ability of the cells to secret matrix molecules, including type I (COL1A1) and III (COL3A1) collagens, and fibronectin. Furthermore, collagen-modifying proteins such as SPARC and TG2 were also detected. The changes to the levels of CO-

TSSC3 Suppresses TGF- $\beta 1$-Induced Renal Fibrosis tive proteins on the Western blots was normalized using the level of GAPDH. OE-NC: treatment with negative control plasmids; TSSC3-OE: treatment with TSSC3-overexpressing plasmids; TGF: treatment with $50 \mathrm{ng} / \mathrm{mL}$ TGF- $\beta 1$; and $740 \mathrm{Y}-\mathrm{P}$ : treatment with 500 $\mu \mathrm{g} / \mathrm{mL} 740 \mathrm{Y}-\mathrm{P}$. Data are shown as the mean with the SD. ${ }^{* *} p<$ $0.01,{ }^{* * *} p<0.001$. ns, not significant; TSSC 3 , tumor-suppressing STF cDNA 3; ECM, extracellular matrix; TGF- $\beta 1$, transforming growth factor beta 1 ; MF, myofibroblast; $\alpha$-SMA, $\alpha$-smooth muscle actin; GAPDH, glyceraldehyde-3-phosphate dehydrogenase.

L1A1, COL3A1, fibronectin, SPARC, and TG2 showed similar trend to that of $\alpha$-SMA, which was upregulated by TGF- $\beta 1$ treatment and downregulated by TSSC 3 overexpression (Fig. 5a, b). Finally, the comparison between TGF$\beta 1+$ TSSC $3-O E+740 Y-P$ and TGF- $\beta 1+$ TSSC $3-O E$ indicated that TSSC 3 attenuated the TGF- $\beta 1$-induced contractile force and matrix production in MFs through the PI3K/Akt signaling pathway (Fig. 5).

\section{Discussion}

In the present study, we identified TGF- $\beta 1$ as an inducer of anoikis resistance of MFs, which is capable of promoting renal fibrosis. The results showed that treatment of 
HK- 2 cells with TGF- $\beta 1$ could not only induce their differentiation into MFs, which was confirmed by upregulation of $\alpha$-SMA and downregulation of villin, but also alleviated the anoikis of MFs induced by RGD-containing peptide treatment or culturing in ultralow attachment culture plates. TGF- $\beta 1$ is a critical factor that affects the progression of fibrotic diseases, whose expression is significantly upregulated in fibrotic tissues, such as the kidney, lung, and liver [25]. TGF- $\beta 1$ affects cell growth, apoptosis, and differentiation; increases the production of the ECM; maintains the viability of fibroblasts; reduces collagen degradation; and thus promotes the fibrotic process [25]. Mechanistic investigations indicated that TGF- $\beta 1$ might promote renal fibrosis by regulating downstream signaling pathways such as the Smad and p53 pathways $[26,27]$ or downstream functions such as macrophage infiltration and epithelial-to-mesenchymal transition [28,29]. By contrast, anoikis is a unique pattern of programmed cell death that acts as an important shield against tumor metastasis and plays a critical role in the clearance of circulating tumor cells. Studies have demonstrated that the acquisition of anoikis resistance is responsible for promoting tumor metastasis. Our previous work also revealed the inhibitory effects of eliminating anoikis resistance on the metastasis of osteosarcoma [23]. Although most research concerning anoikis has focused on malignant tumors, Kim's group found that invasive breast cancer cells induced an anoikisresistant phenotype of MFs during tissue remodeling, the mechanism of which might involve the upregulation of laminin-332 [30]. Therefore, anoikis resistance might be implicated in the acquisition of migratory and invasive characteristics of MF function. In this respect, Horowitz et al. [12] demonstrated that the stimulation of MFs by TGF- $\beta 1$ could confer an anoikis-resistant phenotype by activating focal adhesion kinase and Akt signaling, thus promoting lung fibrosis.

The present study identified TSSC3, which has been extensively investigated as a tumor suppressor in human cancers, especially osteosarcoma, as a suppressor of renal fibrosis, which functions by inhibiting TGF- $\beta 1$-induced anoikis resistance of HK-2 cells or MFs. Among the imprinted genes identified till now, TSSC 3 is the only one related to apoptosis. Our group conducted a series of studies concerning the role and function of TSSC 3 in the development of osteosarcoma and its potential as a prognostic indicator [21]. We first illustrated the involvement of TSSC3 in osteosarcoma growth by inhibiting stemness and apoptosis of osteosarcoma-initiating cells [20], thus acting as a tumor suppressor. Further exploration showed that the inhibition of tumor growth by TSSC 3 overexpression also enhanced the therapeutic effects against osteosarcoma [31]. The repression of osteosarcoma by TSSC 3 could be attributed to the suppressed self-renewal of tumor stem cells and the downregulation of Nanog expression by regulating the Src/Akt signaling pathway [32]. By contrast, we also demonstrate that TSSC 3 overexpression could block epithelial-to-mesenchymal transition and osteosarcoma metastasis, which might have resulted from the regulation of the Wnt/GSK-3 $\beta / \beta$-catenin/Snail signaling pathway or autophagy by the PI3K/Akt/mTOR signaling pathway [22, 33]. More importantly, it was demonstrated that TSSC3, together with RAN-binding protein 9, attenuated anoikis resistance of osteosarcoma cells and prevent tumor metastasis [23]. Consistently, in the present study, we revealed that overexpression of TSSC 3 in HK-2 cells could partially reverse TGF- $\beta 1$-induced anoikis resistance and cell growth, which was confirmed by the increased activities of caspase 3 and caspase 9, the upregulated levels of Bax and cytosolic cytochrome $c$, and the downregulated level of Bcl-2. TSSC 3 is implicated in mitochondrial-mediated apoptosis [20]. Therefore, we speculated that the mitochondria-initiated pathway was involved in TSSC3-facilitated anoikis in TGF- $\beta 1$-induced MFs. Moreover, the ectopic expression of TSSC 3 also mitigated the TGF- $\beta 1$-induced promotion of cell migration and invasion, which was consistent with the downregulation of MMP-2 and MMP-9. Additionally, TSSC3 further alleviated the TGF- $\beta 1$-induced enhancement of contractile force and the secretion of ECM proteins, including COL1A1, COL3A1, fibronectin, SPARC, and TG2. All these results identified TSSC3 as a suppressor of renal fibrosis.

The PI3K/Akt signaling pathway is one of the most thoroughly studied signaling pathways to date. It has distinct regulatory effects on cell proliferation, apoptosis, migration, invasion, and other phenotypes, and plays an important role in tumor progression and deterioration $[34,35]$. Moreover, accumulating evidence has proven that the PI3K/Akt signaling pathway has significant regulatory effects on tissue fibrosis through regulating ECM degradation and the activities of fibrosis-related cells, such as fibroblasts $[36,37]$. Our group also demonstrated the relationship between anoikis and the PI3K/Akt signaling pathway in osteosarcoma, which mediated the regulatory effects of ID1 and TSSC 3 on anoikis and tumor metastasis $[23,38]$. In the present study, the involvement of the PI3K/Akt signaling pathway in the regulation of renal fibrosis by TGF- $\beta 1$ and TSSC 3 was evaluated. The results demonstrated that treatment with TGF- $\beta 1$ enhanced the activity of the PI3K/Akt pathway while improving anoikis and TSSC 3 overexpression induced op- 
posite effects. Treatment with the PI3K/Akt inhibitor (LY294002) showed similar inhibitory effects on TGF$\beta 1$-induced anoikis resistance of MFs overexpressing TSSC3. Moreover, enhanced activation of PI3K/Akt by the activator (740Y-P) showed a similar trend to TGF- $\beta 1$ stimulation in the regulation of anoikis resistance, cell growth, cell migration, cell invasion, ECM production, and cell contraction, which could weaken the alleviation by TSSC 3 overexpression on TGF- $\beta 1$-induced effects.

\section{Conclusion}

Our study demonstrated that TGF- $\beta 1$ could confer an anoikis-resistant phenotype on MFs to promote renal fibrosis, which was mediated by the PI3K/Akt signaling pathway and could be alleviated by the expression of TSSC3.

\section{Statement of Ethics}

Our study does not involve any human subjects and ethics statement is not applicable.

\section{Conflict of Interest Statement}

The authors have no conflicts of interest to declare.

\section{Funding Sources}

This study was financially supported by the Natural Science Foundation of Chongqing (No. cstc2020jcyj-msxmX0013).

\section{Author Contributions}

H.D. designed this study. F.X., X.L., and Y.C. completed all the experiments. H.D. and F.X. conducted the data collection and analysis. H.D. edited the manuscript. All the authors have agreed to the submission of the manuscript.

\section{Data Availability Statement}

All data generated or analyzed during this study are included in the published article.

\section{References}

1 Nogueira A, Pires MJ, Oliveira PA. Pathophysiological mechanisms of renal fibrosis: a review of animal models and therapeutic strategies. In Vivo. 2017;31:1-22.

2 Sun YB, Qu X, Caruana G, Li J. The origin of renal fibroblasts/myofibroblasts and the signals that trigger fibrosis. Differentiation. 2016;92:102-7.

3 Meng XM, Nikolic-Paterson DJ, Lan HY. TGF- $\beta$ : the master regulator of fibrosis. Nat Rev Nephrol. 2016;12:325-38.

4 Humphreys BD. Mechanisms of renal fibrosis. Annu Rev Physiol. 2018;80:309-26.

5 Tang PM, Zhang YY, Mak TS, Tang PC, Huang XR, Lan HY. Transforming growth factor- $\beta$ signalling in renal fibrosis: from Smads to non-coding RNAs. J Physiol. 2018; 596:3493-503.

6 Isaka Y. Targeting TGF- $\beta$ signaling in kidney fibrosis. Int J Mol Sci. 2018;19:2532.

7 Matsumoto K, Xavier S, Chen J, Kida Y, Lipphardt M, Ikeda R, et al. Instructive role of the microenvironment in preventing renal fibrosis. Stem Cells Transl Med. 2017;6:992-1005.

8 Taddei ML, Giannoni E, Fiaschi T, Chiarugi P. Anoikis: an emerging hallmark in health and diseases. J Pathol. 2012;226:380-93.

9 Paoli P, Giannoni E, Chiarugi P. Anoikis molecular pathways and its role in cancer progression. Biochim Biophys Acta. 2013;1833: 3481-98.
10 Wang YN, Zeng ZL, Lu J, Wang Y, Liu ZX, He MM, et al. CPT1A-mediated fatty acid oxidation promotes colorectal cancer cell metastasis by inhibiting anoikis. Oncogene. 2018;37: 6025-40.

11 Simpson CD, Anyiwe K, Schimmer AD. Anoikis resistance and tumor metastasis. Cancer Lett. 2008;272:177-85.

12 Horowitz JC, Rogers DS, Sharma V, Vittal R, White ES, Cui Z, et al. Combinatorial activation of FAK and AKT by transforming growth factor-betal confers an anoikis-resistant phenotype to myofibroblasts. Cell Signal. 2007; 19:761-71.

13 Ishida M, Moore GE. The role of imprinted genes in humans. Mol Aspects Med. 2013;34: $826-40$.

14 Barlow DP, Bartolomei MS. Genomic imprinting in mammals. Cold Spring Harb Perspect Biol. 2014;6:a018382.

15 Jacobs DI, Mao Y, Fu A, Kelly WK, Zhu Y. Dysregulated methylation at imprinted genes in prostate tumor tissue detected by methylation microarray. BMC Urol. 2013;13:37.

16 Kim J, Bretz CL, Lee S. Epigenetic instability of imprinted genes in human cancers. Nucleic Acids Res. 2015;43:10689-99.

17 Joyce JA, Schofield PN. Genomic imprinting and cancer. Mol Pathol. 1998;51:185.
18 Lee MP, Feinberg AP. Genomic imprinting of a human apoptosis gene homologue, TSSC3. Cancer Res. 1998;58:1052.

19 Altinoz MA, Elmaci I, Ince B, Ozpinar A, Sav AM. Hemoglobins, hemorphins, and 11p15.5 chromosomal region in cancer biology and immunity with special emphasis for brain tumors. J Neurol Surg Part A. 2016;77:247-57.

20 Huang Y, Dai H, Guo QN. TSSC3 overexpression reduces stemness and induces apoptosis of osteosarcoma tumor-initiating cells. Apoptosis. 2012;17:749-61.

21 Wang $\mathrm{X}, \mathrm{He} \mathrm{H}$, Zhang K, Peng W. The expression of TSSC 3 and its prognostic value in patients with osteosarcoma. Biomed Pharmacother. 2016;79:23-6.

22 Lv YF, Dai H, Yan GN, Meng G, Zhang X, Guo QN. Downregulation of tumor suppressing STF cDNA 3 promotes epithelial-mesenchymal transition and tumor metastasis of osteosarcoma by the Wnt/GSK-3 $\beta / \beta$-catenin/ Snail signaling pathway. Cancer Lett. 2016; 373:164-73.

23 Dai H, Lv YF, Yan GN, Meng G, Zhang X, Guo QN. RanBP9/TSSC3 complex cooperates to suppress anoikis resistance and metastasis via inhibiting Src-mediated Akt signaling in osteosarcoma. Cell Death Dis. 2016;7: e2572. 
24 Cao Y, Deng C, Townsend CM, Ko TC. TGFbeta inhibits Akt-induced transformation in intestinal epithelial cells. Surgery. 2006;140: $322-9$.

25 Kim KK, Sheppard D, Chapman HA. TGF- $\beta 1$ signaling and tissue fibrosis. Cold Spring Harb Perspect Biol. 2018; 10:a022293.

26 Higgins SP, Tang Y, Higgins CE, Mian B, Zhang W, Czekay RP, et al. TGF- $\beta 1 /$ p53 signaling in renal fibrogenesis. Cell Signal. 2018; 43:1-10.

27 Loboda A, Sobczak M, Jozkowicz A, Dulak J. TGF- $\beta 1 /$ Smads and miR-21 in Renal Fibrosis and Inflammation. Mediators Inflamm. 2016; 2016:8319283.

28 Hu N, Duan J, Li H, Wang Y, Wang F, Chu J, et al. Hydroxysafflor yellow a ameliorates renal fibrosis by suppressing TGF- $\beta 1$-induced epithelial-to-mesenchymal transition. PLoS One. 2016;11:e0153409.

29 Chung S, Overstreet JM, Li Y, Wang Y, Niu A, Wang $\mathrm{S}$, et al. TGF- $\beta$ promotes fibrosis after severe acute kidney injury by enhancing renal macrophage infiltration. JCI Insight. 2018;3: e123563.
30 Kim BG, Gao MQ, Choi YP, Kang S, Park HR, Kang KS, et al. Invasive breast cancer induces laminin-332 upregulation and integrin $\beta 4$ neoexpression in myofibroblasts to confer an anoikis-resistant phenotype during tissue remodeling. Breast Cancer Res. 2012;14:R88.

31 Dai H, Huang Y, Li Y, Meng G, Wang Y, Guo QN. TSSC3 overexpression associates with growth inhibition, apoptosis induction and enhances chemotherapeutic effects in human osteosarcoma. Carcinogenesis. 2012;33:3040.

32 Yan GN, Tang XF, Zhang XC, He T, Huang YS, Zhang X, et al. TSSC3 represses self-renewal of osteosarcoma stem cells and Nanog expression by inhibiting the Src/Akt pathway. Oncotarget. 2017;8:85628-41.

33 Zhao GS, Gao ZR, Zhang Q, Tang XF, Lv YF, Zhang ZS, et al. TSSC3 promotes autophagy via inactivating the Src-mediated PI3K/Akt/ mTOR pathway to suppress tumorigenesis and metastasis in osteosarcoma, and predicts a favorable prognosis. J Exp Clin Cancer Res. 2018;37:188.
34 Vara JÁF, Casado E, de Castro J, Cejas P, Belda-Iniesta C, González-Barón M, et al. PI3K/ Akt signalling pathway and cancer. Cancer Treatment Reviews. 2004;30(2):193-204.

35 Song M, Bode AM, Dong Z, Lee MH. AKT as a therapeutic target for cancer. Cancer Res. 2019;79(6):1019.

36 Hsu HS, Liu CC, Lin JH, Hsu TW, Hsu JW, Su $\mathrm{K}$, et al. Involvement of ER stress, PI3K/AKT activation, and lung fibroblast proliferation in bleomycin-induced pulmonary fibrosis. Sci Rep. 2017;7:14272.

37 Cui LH, Li CX, Zhuo YZ, Yang L, Cui NQ, Zhang SK. Saikosaponin d ameliorates pancreatic fibrosis by inhibiting autophagy of pancreatic stellate cells via $\mathrm{PI} 3 \mathrm{~K} / \mathrm{Akt} / \mathrm{mTOR}$ pathway. Chem Biol Interact. 2019;300:1826.

38 Zhao GS, Zhang Q, Cao Y, Wang Y, Lv YF, Zhang ZS, et al. High expression of ID1 facilitates metastasis in human osteosarcoma by regulating the sensitivity of anoikis via PI3K/ AKT depended suppression of the intrinsic apoptotic signaling pathway. Am J Transl Res. 2019;11:2117-39. 